\title{
Wireless Communications for the Hospital of the Future: Requirements, Challenges and Solutions
}

\author{
Iqrar Ahmed ${ }^{1}$ [ $\cdot$ Heikki Karvonen ${ }^{1} \cdot$ Timo Kumpuniemi $^{1} \cdot$ Marcos Katz $^{1}$
}

Received: 28 February 2019 / Revised: 28 June 2019 / Accepted: 10 October 2019 / Published online: 28 October 2019

(c) The Author(s) 2019

\begin{abstract}
In this conceptual paper, we discuss the concept of hospital of the future $(\mathrm{HoF})$ and the requirements for its wireless connectivity. The HoF will be mostly wireless, connecting patients, healthcare professionals, sensors, computers and medical devices. Spaces of the HoF are first characterized in terms of communicational performance requirements. In order to fulfil the stringent requirements of future healthcare scenarios, such as enhanced performance, security, safety, privacy, and spectrum usage, we propose a flexible hybrid optical-radio wireless network to provide efficient, high-performance wireless connectivity for the HoF. We introduce the concept of connected HoF exploiting reconfigurable hybrid optical-radio networks. Such a network can be dynamically reconfigured to transmit and receive optical, radio or both signals, depending on the requirements of the application. We envisage that HoF will consist of numerous communication devices and hybrid optical-radio access points to transmit data using radio waves and visible light. Light-based communications exploit the idea of visible light communications (VLC), where solid-state luminaries, white light-emitting diodes (LEDs) provide both room illumination as well as optical wireless communications (OWC). The hybrid radio-optical communication system can be used in principle in every scenario of the HoF. In addition to the hybrid access, we also propose a reconfigurable opticalradio communications wireless body area network (WBAN), extending the conventional WBAN to more generic and highly flexible solution. As the radio spectrum is becoming more and more congested, hybrid wireless network approach is an attractive solution to use the spectrum more efficiently. The concept of HoF aims at enhancing healthcare while using hospital resources efficiently. The enormous surge in novel communication technologies such as internet of things (IoT) sensors and wireless medical communications devices could be undermined by spectral congestion, security, safety and privacy issues of radio networks. The considered solution, combining optical and radio transmission network could increase spectral efficiency, enhancing privacy while reducing patient exposure to radio frequency (RF). Parallel radio-optical communications can enhance reliability and security. We also discuss possible operation scenarios and applications that can be introduced in $\mathrm{HoF}$ as well as outline potential challenges.
\end{abstract}

Keywords Hospital of the future $\cdot$ Reconfigurable networks · Visible light communications · Hybrid wireless networks · Optical wireless communications $\cdot$ Wireless body area networks

\section{Introduction}

In recent years, the concept of connected hospital of the future $(\mathrm{HoF})$ has received considerable attention by the healthcare society [1-3]. The paradigm of HoF comprises

Iqrar Ahmed

iqrar.ahmed@oulu.fi

Heikki Karvonen

heikki.karvonen@oulu.fi

1 Centre for Wireless Communications (CWC), University of Oulu, Oulu, Finland of a hospital connected in such a way that state-of-the-art healthcare services could be provided to each patient regardless of the limited resources and hospital boundaries [4]. Oulu University Hospital in Finland is developing its own HoF concept called Future Hospital OYS 2030 [5]. The goal of this initiative is to define and build a healthcare facility equipped with modern technologies, using up-to-date standards to efficiently distribute and utilize hospital resources, ultimately providing cost effective and state-of-the-art healthcare to everyone. The HoF concept also includes home or remote healthcare facilities [6, 7], allowing realtime monitoring of patients [8], remote control of robots 
[9], implants or actuators, etc. Truly connected healthcare can only be achieved if every stakeholder of the HoF is connected to a communication highway where the hulk of information between patients, equipment and healthcare personnel is efficiently and timely delivered $[10,11]$.

Wireless communications have an utmost important role in the HoF as the goal of the concept is to connect all key stakeholders. Radio frequency (RF) communications have been the preferred technology to provide a seamless communication highway for connecting these stakeholders. However, modern healthcare environments pose some challenges to conventional radio communications. In fact, requirements for security and privacy, interference generation and tolerance, as well as safety defy radio systems. In addition, spectrum congestion and energy efficiency are also clear challenges for radio-based communications systems [12]. All these issues affect the attractiveness of radio in healthcare scenarios. In the past decade, visible light communications (VLC) technology has rapidly emerged as a viable way to transmit information wirelessly. VLC exploits the lighting infrastructure of solid-state light sources (e.g., white light-emitting diodes) to provide light-based wireless connectivity. There has been a growing interest of utilizing VLC in hospitals, in $[13,14]$ authors have demonstrated the utilization of VLC in hospital to transmit data and have discussed the additional benefits of VLC including radiation free, secure and fairly inexpensive communication. VLC has proven effective technology for potential healthcare applications such as remote health monitoring [8] and controlling autonomous robots [9]. There are many proven features that make VLC feasible for healthcare scenarios. Examples include indoor localization too, in [15] an indoor localization with almost no light is demonstrated that could be helpful in positioning not only the robots but to localize the patients within the hospital.

A hospital habitat comprises of people (e.g., patients, medical staff, visitors), processes (e.g., diagnosis, treatment, management of people, spaces, material, equipment, time, etc.), technology, and premises. The concept of hospital is in continuous development, aiming ultimately at providing the best possible healthcare to patients; balanced, safe and productive working conditions to hospital staff; and comfort to visitors, while operating in an efficient, cost-effective and sustainable manner. The role of technology is central in shaping future of healthcare as technology can tackle tedious tasks e.g., vital signs monitoring, to ease clinicians [16]. The rapid advances in key technologies related to hospitals are expected to have a huge impact on the HoF. Hospital can be of many sizes and capabilities, from huge university hospital complexes covering virtually any aspect of medical care till small specialized hospital units, and from public healthcare institutions to private hospitals. In this paper, we assume a large hospital with the most common healthcare scenarios and other required facilities. The concept of large hospital does not only consider a centralized medical care in a facility located at a certain location, but also distributed out-hospital services made available in, e.g., patients' homes. Hospitals are in a constant state of innovation and adaptation [17]. Novel technologies and the need to improve the healthcare process, increase operations efficiency and reduce costs create such needs. Authors in [18] identify key characteristics of the HoF, including;

a. HoFs will be open and distributed organizations,

b. HoFs will become innovation centers,

c. HoFs will offer integrated care with process-oriented teams, and

d. HoF will be connected hospitals.

These characteristics encourage the use of wireless communications in the HoF to provide connectivity to medical, management and operational staff, patients, visitors, hospital equipment, sensors as well as to control the multiple processes needed to manage the hospital. Hospitals are generally designed to be patient centric. When designing hospital, several trends in healthcare have been identified, and one can expect to see them widely implemented in HoF. These include;

a. clear division between inpatient and outpatient healthcare, aiming to maximize outpatient care not only due to cost issues but also because of its important advantages (e.g., reducing hospital-acquired infection risks, better patient care and satisfaction, etc.),

b. 24/7 reliable monitoring as the driving force of remote healthcare or telemedicine,

c. in addition to healthcare, HoF should also focus on prevention and wellbeing, and

d. real-time monitoring and data collection of management processes to create rapid and optimized responses.

Technology is probably the most important enabler of the HoF concept. The HoF will be digital, wireless and exploit widely artificial intelligence (AI) and big data [19, 20]. Other technologies expected to be exploited in the $\mathrm{HoF}$ concept may include medical robots, automation, wearable devices, positioning, augmented/ virtual reality (AR/VR), 3D printing, blockchain, security, smart lighting, etc. It is interesting to note that wireless communications is virtually related to all these hospital technologies, either as an enabling or support technology.

After reviewing the concept of connected $\mathrm{HoF}$ and discussing its requirements and challenges, we propose a hybrid optical-radio network as the key connectivity infrastructure for hospitals in the future. The proposed hybrid network combines the advantages of both radio and optical 
approaches to create a highly flexible, high performance, highly secure, private and safe communication network suitable for all demanding healthcare scenarios of the HoF. This paper is organized as follows: Sect. 2 discusses wireless communication systems for the HoF, while Sect. 3 introduces their communicational requirements. The proposed wireless communication solutions for the $\mathrm{HoF}$ is presented in Sect. 4. Section 5 introduces the challenges associated with the communication systems of HoF. Finally, Sect. 6 contains the discussions and conclusions of this study.

\section{Wireless Communications for Future Hospitals}

As discussed, wireless communications are essential for the $\mathrm{HoF}$ as flexible and scalable connectivity is necessary to interconnect all the stakeholders inside and outside the premises. Other important characteristics for the wireless network include data throughput, reliability, latency, security and privacy, cost-efficiency and seamless integration. How stringent are these performance figures depends on the type of scenario and particular application considered. Generally, a hospital contains number of communication devices where the electronic health records, real-time sensor data and other important information flows among those. Radio is today the de facto technology for wireless networking and radiobased wireless networks are exclusively used in hospitals to transmit wirelessly information. Radio communications is a well-developed technology, flexible, inexpensive and widely standardized. Two major approaches are used, namely centralized cellular-type of networks to provide wide area coverage, as well as short-range communications, providing connectivity over short distances, typically shorter than few hundred meters. Certainly, these networks are in practice combined in many ways [21]. The widely popular wireless local area networks (e.g., Wi-Fi), wireless sensor networks (WSN) [22], wireless personal area networks (WPAN) [23] and wireless body area networks (WBAN) [24] are examples of short-range wireless networks technologies that can be used in the HoF. Moreover, cellular technology can also be exploited, for instance deploying 5G (and beyond) picocells within the hospital and other healthcare facilities. Novel communications paradigms such a internet of things (IoT) [25] and internet of medical things (IoMT) will certainly find use within the HoF. One can expect that the number of stakeholders connected wirelessly in the HoF will increase substantially. In an in-building network, the service is provided and managed inside the premises of the hospital. Hospital connectivity can also be provided in an out-building manner, where base stations outside the hospital.

Figure 1 depicts the different wireless and mobile networks providing connectivity to the HoF. Most of these network technologies are based on the use of radio. Despite of its advantages, there are certain areas where operation of radio systems is not optimum, particularly in the complex and demanding scenarios expected to be encountered in the HoF. Since there are multiple radio technologies operating in the same environment, the challenge is to mitigate against interference between wireless systems, exposure to electromagnetic radiation by patients and hospital staff, spectrum congestion and others.

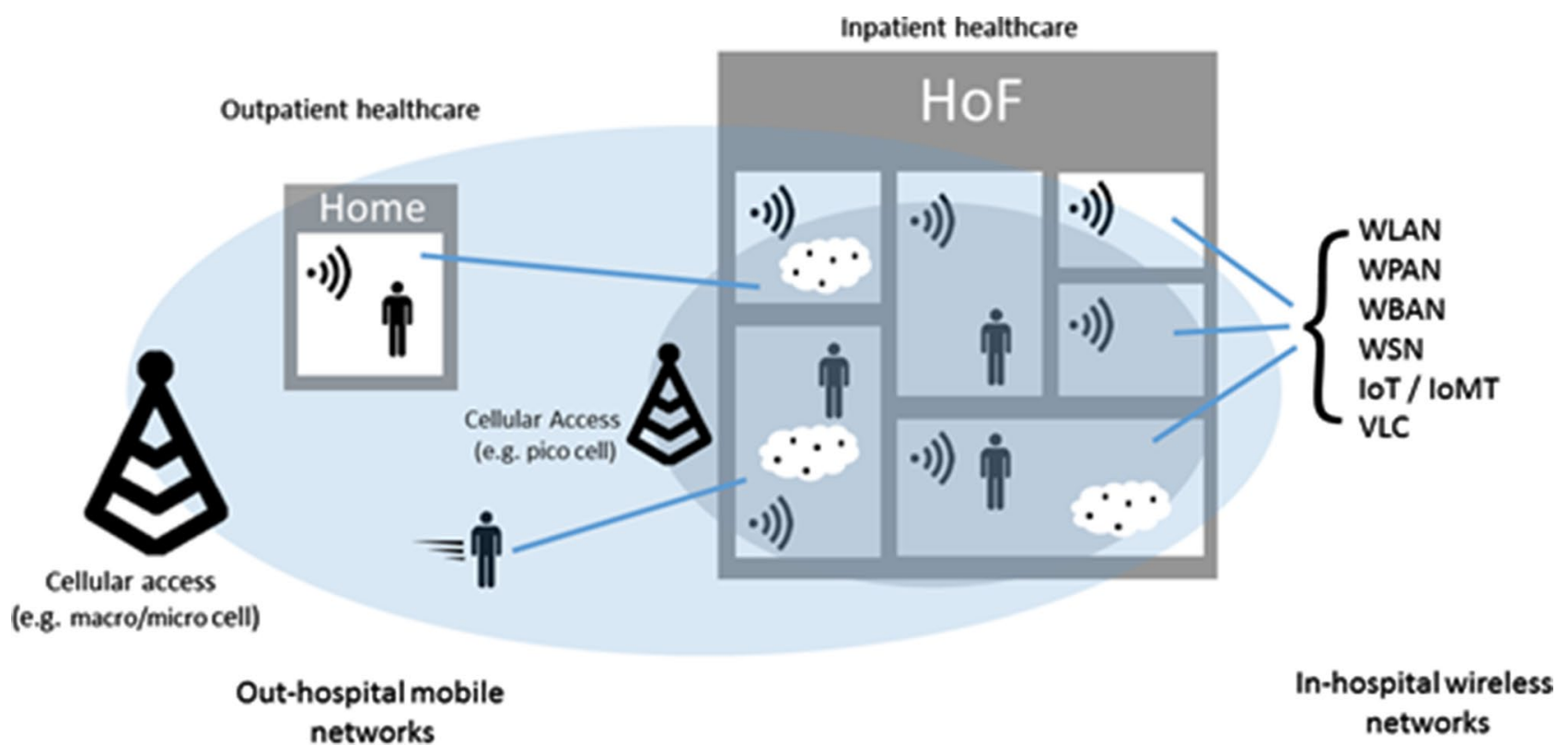

Fig. 1 Wireless and mobile networks for the HoF 
Light-based communications, such as VLC is also a highly attractive communication technology for healthcare environments. VLC exploits modern solid-state light sources, i.e., white light emitting diodes (LEDs) to create, in addition to illumination, an optical transmission capable to convey information, in the same way that short-range radio transmissions do. The lighting infrastructure can easily provide a downlink channel, while, uplink connectivity can be supported by installing radio receives on the ceiling or other suitable place. For hybrid system, it is more practical to implement ulpink using infrared communications. Interestingly, the key advantages of VLC are precisely in those areas where radio systems are weak, for instance security, interference tolerance and generation, electromagnetic exposure point of view. Thus, radio and optical systems are highly complementary to each other. Combining radio and optical wireless systems has been described and exploited in $[26,27]$, where authors propose the use of a reconfigurable radio-optical network. Optical wireless communications (OWC) complement radio and together can create a highly flexible and high-performance network capable to fulfill the stringent requirement of future hospitals. This hybrid wireless network is proposed and further studied in this paper.

\section{Communicational Requirements for the HoF}

In this section, we discuss key requirements that are viable for communication technologies at the HoF. As introduced above, there are various potential applications enabled by wireless communication at future hospitals. Requirements strongly depend on the application. In turn, applications depend on the hospital under consideration. A university hospital with all the specialist branches of medicine, with the most advanced research and treatment methods, and with large amount of patients and medical staff concentrated into a large building complex sets its own requirements. A smaller hospital unit intended for e.g., treatment of elderly or a specialised hospital for the most demanding medical procedures intended for children may define quite different applications and wireless communication requirements. Furthermore, if the medical services are decentralized to patients living in their residences, yet different kind of demands will arise. Here we focus on the communicational requirements which are most critical for hospital applications.

The number of wireless devices is increasing very rapidly, and the radio frequency bands are becoming very crowded. In $[28,29]$ authors found that widely used frequency band $2.4 \mathrm{GHz}$ includes many different wireless technologies and they are creating harmful interference to each other. For example, in [30] it was found that the popular Bluetooth low energy (BLE) technology will suffer from interference under a worst-case interference scenario created by ZigBee-based IoT devices. The large and eclectic wireless node base in future hospitals needs to operate without any interference between nodes. Therefore, it is crucial to support co-existence of different communication technologies in future hospital solutions. Optical communication is a viable way to decrease the amount of radio-based communication at crowded frequency bands. The coexistence of optical and RF communication will decrease the spectrum congestion and reduce possible interference problems. Furthermore, such an approach will improve reliability and reduce communication latency.

In critical medical applications, such as intensive care or intensified observation, communication reliability, i.e., dependability, and low latency are highly important since patients health critically depends on it. In static medical measurement scenarios (e.g. operation room or bedside measurements), optical communication can be used to enable reliable data transfer with low latency since the available bandwidth is wide at visible light frequencies. Ultra-low latency and high reliability is required also e.g., in robot surgery. Wide bandwidth of VLC enables very high high data rates which is required by complex medical examinations and procedures, such as computer tomography used together with robot aided operations on the most demanding surroundings as, e.g., brains. Energy efficiency is important to enable long duration operation for battery-powered sensor devices, e.g., in WBAN and IoT nodes. VLC provide a viable way to reduce the RF nodes communication needs which enables more energy efficient operation for them. From the overall energy consumption point of view, the VLC allows a joint design with RF communications aiming at very high energy efficient. VLC is energy efficient due to its nature to provide lighting and communication at the same time. The WBAN sensors may often be battery operated. The adoption of VLC also provides a convenient way to use the existing light to charge the batteries of the sensors in the network, e.g., energy harvesting. Many applications exploit location information of humans and devices equipped with communication transceivers. Indoor localization at hospitals cannot rely on global navigation satellite systems (GNSS), therefore localization is usually done using local signals. For example, BLE technology is used in localization systems, which requires installation of anchor nodes around the hospital space. Localization accuracy depends on the signal bandwidth and therefore visible light communication could provide more accurate solution in comparison to current solutions. Furthermore, visible light localization system would take advantage of the existing lighting infrastructure leading to cost- and energy-efficient solution. Combining radio and light-based localization is expected to improve remarkably flexibility and accuracy. 


\subsection{Communicational Requirements for Different Hospital Spaces}

Communicational requirements are discussed in this section by considering the most relevant hospital spaces. Sprow has characterized hospital with five main spaces as inpatient care, ambulatory care diagnostic and treatment, support services and public spaces [31]. In-patient care is carried out in small to medium size comfortable places, e.g., patient wards, where the time scale of patient staying is measured in days. Ambulatory care are walk-in spaces for short-term care, with staying time is measured in terms of hours. Diagnostic and treatment spaces complement the healthcare of the two first spaces. Diagnostics includes for instance magnetic resonance imaging (MRI) and computerized tomography (CT) scans, laboratory services, ultrasound imaging, electroencephalography (EEG), electrocardiogram (ECG), nuclear medicine and others. Treatment space focus on emergency care, surgery, endoscopy, biopsy, etc. Support services include services for hospital staff, patients, visitors, such as staff facilities, education and training rooms, material supply, pharmacies, kitchen, housekeeping, maintenance, and others. Public spaces, such a waiting rooms, 331 canteens are also important social premises of a hospitals, used by many people. In addition to these in-hospital spaces, outpatient health care needs to be considered in the HoF model. Here, two important out-hospital cases are described, namely care at home and care on the move. In principle, wireless connectivity is needed in each of these spaces.

Table 1 depicts the expected requirements for wireless connectivity in the considered HoF spaces. The values in Table 1 ranges between 1 and 5, where 1 is very-low and 5 means very-high. We can see that high data rate is required when treating the patients within the hospital or in emergency, i.e., ambulatory care or support services and the latency can be moderate to very low. On the other hand, these requirements for outpatient care are somewhat opposite. The reliability and security requirements are high since a loss of data could result in wrong diagnosis and results could be devastating. Breach of personal health data could 349 be a potentialthreat to the patient. Mobility is usually restricted when patients are admitted or undergoing emergency treatment, very-low mobility is required in these cases for wireless communications, especially when the data is transmitted or received optically line-of-sight (LOS) communication is necessary to avoid any link breakages. While for outpatient high mobility is allowed for patients, to avoid data loss offloading i.e., data stored and transmitted when device comes in contact to an optical or radio access point 357 (AP) could be used. For public spaces and outpatient scenarios the interference detection is kept to low since it is obvious that link breakage or degradation may occur due to many reasons e.g., if patient moves away from optical hotspot or light is blocked by someone. But for inpatient care and other cases where the patient is not moving, a fairly high sensitivity is required. Sensitivity means that people are less exposed to RF radiations, that is why a fairly moderate sensitive wireless network is required. The latency requirements for inpatient application such as diagnostic/ treatment room is ranging from low to moderate based on the undergoing procedure. For diagnostics i.e., CT and MRI a moderate latency is sufficient but for procedures undergoing in operation theatres, very low latency is required. Finally, users network capacity requirements could be diverse, i.e., ranging low to very high.

Hospital communication systems must enable prioritization of data transmissions, i.e., critical data must be always transmitted using the required data rate, reliability and latency. In medical communication systems, security is highly important from many perspectives, due to nature of the data content, as discussed in $[32,33]$. The data stream must be dependable against any unintentional or intentional interference. From the patients privacy point of view, it must safe from any information leakage to quarters to whom the data is not intended to be distributed. From the safety point of view, the communication must not induce excessive

Table 1 Performance requirements for wireless networks in key HoF spaces (local and remote)

\begin{tabular}{lllllllll}
\hline & Data rate & Reliability & Mobility & Security & Latency & $\begin{array}{l}\text { Sensitivity } \\
\text { (interference) }\end{array}$ & $\begin{array}{l}\text { Sensitivity } \\
\text { (exposure) }\end{array}$ & $\begin{array}{l}\text { Capacity } \\
\text { (supported } \\
\text { devices) }\end{array}$ \\
\hline Inpatient care & & & & & & & & \\
Ambulatory care & $2-5$ & $4-5$ & $1-2$ & $4-5$ & $1-3$ & $3-4$ & $2-4$ & $3-5$ \\
Diagnostic/ treatment & $2-5$ & $4-5$ & $1-2$ & $4-5$ & $1-3$ & $3-4$ & $2-4$ & $2-4$ \\
Support services & $4-5$ & $4-5$ & $1-2$ & $4-5$ & $1-3$ & $3-5$ & $2-4$ & $2-4$ \\
Public spaces & $4-5$ & $4-5$ & $1-3$ & $4-5$ & $1-3$ & $3-5$ & $2-4$ & $3-5$ \\
Outpatient care (at home) & $3-4$ & $3-4$ & $1-3$ & $4-5$ & $2-4$ & $1-2$ & $2-4$ & $3-5$ \\
Outpatient care (on the move) & $1-3$ & $4-5$ & $1-2$ & $4-5$ & $2-4$ & $1-2$ & $2-4$ & $3-5$ \\
\hline
\end{tabular}

5: very-high; 4: high; 3 : moderate, 2 : low; 1: very-low 
electromagnetic radiation to the human tissues, measured by the specific absorption rate (SAR). The adoption of VLC will facilitate all the above-mentioned demands. Firstly, as VLC can typically be focused on a small area, e.g., within one room, the robustness against unintentional or intentional interference is highly diminished. Secondly, as the VLC signal can be limited inside one room, it will not leak or cannot be eavesdropped from, e.g., the neighboring rooms or outside. And thirdly, the usage of VLC will decrease the need for the usage of RF- based communication systems, thus lowering the cumulative SAR load on the patients. Table 2 gives an overview of comparison between RF and VLC, while being widely used commercially RF has security, privacy and exposure issues. On the other hand, the VLC is newly developed technology that has issued such as low coverage areas and need for LOS link etc.

\section{A Communication Network for the Connected HoF}

Over the decades, we have witnessed an enormous development in mobile communication, still the RF is a predominant technology and will most likely continue to be in wireless communications. The favorable aspects of RF i.e., non-line of sight communication, both long- and short-range communications, availability of multiple standards, low cost, etc., have kept this technology attractive for wireless communications. However, associated challenges of security, privacy, and spectrum congestion have grown concerns and have motivated researchers to find complementary alternatives. Interference free reliable data transmission could be challenging with the deployment of IoT where several wireless sensors will be transmitting data at the same time. Spectrum overloading could be a severe hurdle in future communications, particularly in our case where the communication backbone is key stakeholder and foundation to build a HoF. To overcome these challenge, we propose the concept of hybrid optical-radio network. In this chapter, we discuss the our proposed reconfigurable hybrid network, intelligent algorithm for reconfiguration, handover techniques and finally we present potential solutions of proposed network.

\subsection{A Reconfigurable Hybrid Optical-Radio Network for HoF}

We argue that optical wireless network can be embedded to an existing RF backbone, the complementary nature of light could prove to be a viable alternative to RF. The complementary optical and radio networks would not only enhance the redundancy, an efficient use of spectrum would possibly reduce the spectrum congestion or overloading while communicating on both network simultaneously. Indoor applications of hybrid networks have been predominantly studied $[34,35]$, as in [34] an indoor application of hybrid opticalradio network is considered to transmit the indoor traffic to base station using a RF relay. Associated challenges such as maximizing the throughput by selective transmission power have also been simulated to reflect the spectrum efficiency of hybrid networks [35]. Hybrid optical-radio networks are needed to be energy efficient compared to RF in order to meet one of the stringent requirement of HoF, authors in [36] studied hybrid VLC/RF and RF networks. The integration of VLC in RF network is shown energy efficient depending on the LED operating power, with the development of low power LED the efficiency of VLC can be further enhanced. An experimental platform is implemented in [37], to demonstrate the hybrid optical-radio communications and proposed
Table 2 The pros and cons of VLC vs. RF

\begin{tabular}{lll}
\hline & RF & VLC \\
\hline Pros. & Wide coverage & High security \\
& LOS not needed & Decreased co-existence problem \\
& Multiple standards & Very high data throughput support \\
& High dependability for connection & No SAR exposure \\
& Several available commercial technologies & Better energy and cost-efficiency \\
& Relatively low cost & Lower PHY layer latency \\
& & Availability of huge unregulated bandwidth \\
& & Integrated with lighting infrastructure \\
Cons. & Decreased security & Compatible with smart lighting \\
& Decreased privacy & Small coverage/each room needs an access point \\
& Increased SAR exposure & Need LOS for operation for best performance \\
Increased co-existence problem & Mainly indoor limited \\
Lower data rate & Mainly short-range limited \\
Higher PHY layer latency & Commercializating slowly \\
\hline
\end{tabular}


suitable for communications purposes in sensitive areas and in different media such as space, air, ground, and ocean. This paper is an extension of our previous works [26, 27].

So far, VLC in healthcare has been used for transmitting bio signals data over optical wireless network [38, 39], the network nodes in these cases are optical transceivers that transmit the modulated bio signals from body sensors. Some other studies show the power line communication (PLC) [13] and VLC applications to construct a data sharing backbone for a hospital. Following the evolution of digital healthcare solutions i.e., medical devices and gadgets to monitor patient's health records, the amount of personal healthcare data has increased enormously. Data is stored both locally and on offshore clouds. Having stored online, the clinicians and the patients can access their data timelessly, the only condition is to have a faster RF wireless link to the cloud.

Here we have extended this generic approach of using a RF wireless network embedded with VLC network to construct a reconfigurable hybrid optical-radio network. The brain of this hybrid network is an intelligent software that can respond to dynamic changes of the network, type or requirements of the healthcare scenario, etc., to select the appropriate network or transmission mode. The software can be reconfigure remotely. The proposed reconfigurable hybrid network comprises of optical and RF transceivers. The optical and RF uplinks (UL) and downlinks (DL) are establish and data transmission is accomplished either solely on optical or radio channels, or the hybrid channels are established combining both optical and radio networks following the certain policies, predefined in reconfiguration software.

The intelligent algorithm is key to attain the maximum throughput from a hybrid system, the complexity of algorithm may increase with network's sensitivity. Resource allocation and optimal network selection are key for an intelligent algorithm in order to maximize the throughput $[40,41]$.

The policies in reconfigurable algorithm are constructed on dynamic changes that may occur during transmission include UL and DL channel conditions for both optical and radio, service requirement, local policies, service user/operator requirements etc. The reconfigurable algorithm selects the optimal UL and DL channels aggregating the dynamic changes in optical and radio channels. Based on the UL and DL selected, we have proposed five modes, the hybrid network can switch between these five modes following the dynamic changes and predefined policies in reconfigurable algorithm.

Mode1. Both downlinks and uplinks are optical.

Mode2. Downlink optical and uplink radio.

Mode3. Downlink radio and uplink optical.

Mode4. Both downlink and uplink are radio.

Mode5. Cooperative mode, both optical and radio transceivers are working simultaneously.
Figure 2 gives an overview of the optical-radio APs forming a mesh. Stakeholders of the HoF are connected to hybrid network, patients and clinicians can retrieve data at their ease. Vital bio sensors data from patients WBAN is transmitted in real-time and can be retrieved.

\subsection{Vertical Handover Techniques}

Embedded benefits in cooperative mode including parallel data transmission, redundancy, increased spectrum etc., make this approach attractive in hospitals. The network selection can be reconfigured dynamically and the selection can be performed according to chosen vertical handover (VHO) technique. From Table 1, we can see that the reliability of wireless network is highly recommended. In case of an unreliable network the potential threat of data loss can threaten the patient's life becasue partial data could result in wrong or misleading diagnosis. To overcome these issues, the hybrid network provides an alternative link to transmit data but the risk of losing data still remains as the network could continue sending data on poor link. Unless we decide on the quality of current link and perform handover in a hybrid network. Gathering channel state information (CSI) for both RF and optical is key to successfully perform handover in aforementioned five operation modes.

Instant vertical handover (IVHO) is a straight technique, that switches the default optical network to RF as soon as the CSI tells the controller that the optical link is unavailable. The controller monitors the optical link continuously and the link reinstate as soon as controller gets acknowledgement. The disadvantage of implementing fast handover is loss of resources in continuous link monitoring and unnecessary switching in short interruptions that could result in packet loss. Dwell time technique gathers the CSI of unavailable optical link in an exponential interval, the objective of the this approach is to conserve resources and avoid unnecessary switching $[42,43]$. As mentioned above the packet loss is inevitable in case of link failure, moreover the frequent handover due to short interruptions could increase the packet loss too, in such scenarios the predictive handover could be more suitable. The attributes such as message length and duration of interruption are used to establish a prediction mechanism where the predicted delays for an instant and dwell handover are compared and handover decision is made on logical set of rules [44].

\subsection{Applications of Hybrid Wireless Networks for HoF}

Though one still needs to visit hospital for complete examination and treatment, remote healthcare could play a viable role in reducing the burden from hospitals. For remote monitoring, WBAN consisted of sensors' mesh usually embedded 


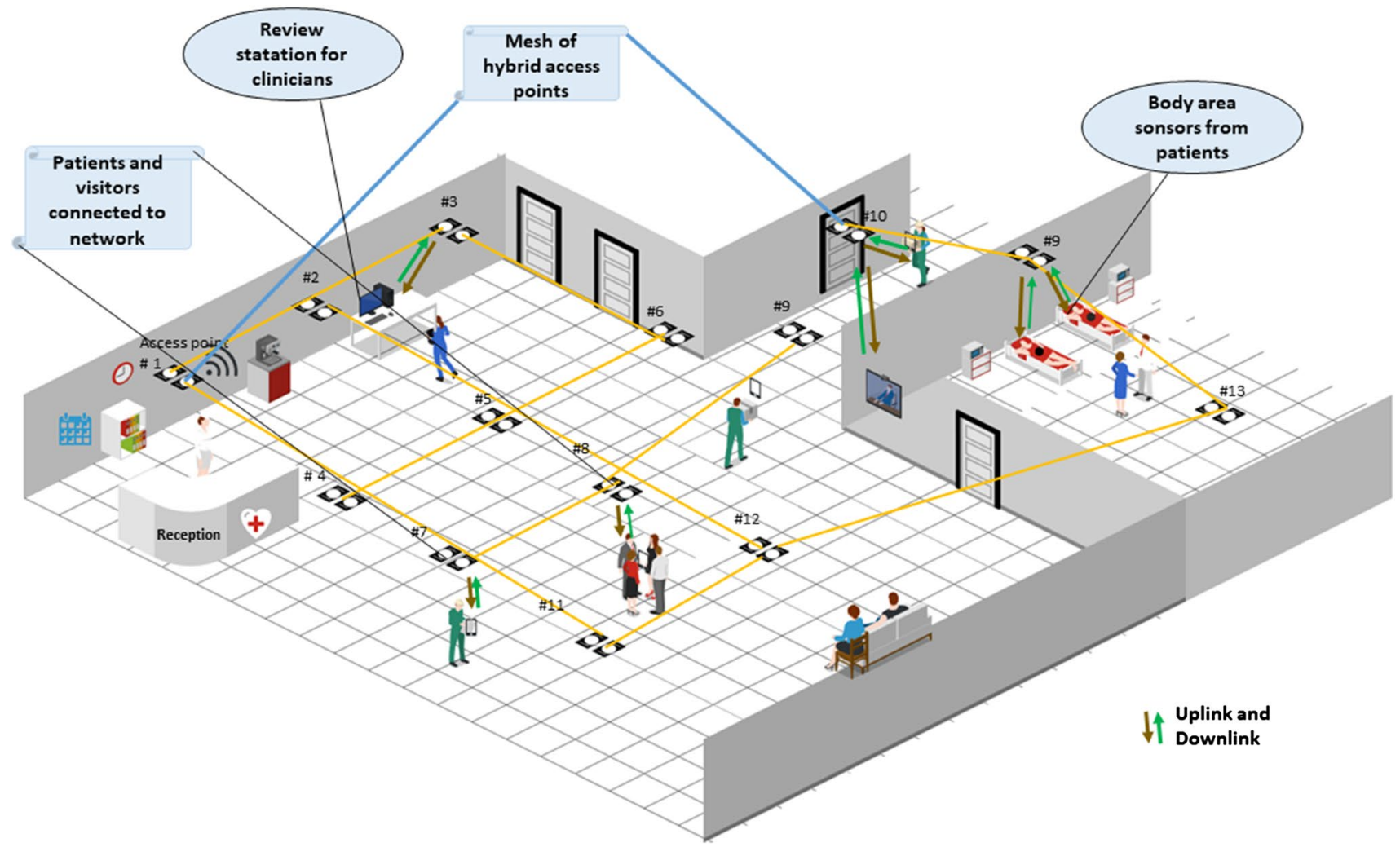

Fig. 2 Stakeholders for HoF. An overview of mesh of hybrid optical-radio access points/hotspots to keep the data flow and provide internet connectivity within a hospital

in a suit is worn by patient and data from the sensor's mesh is collected remotely through RF links. Numerous RF sensors working in a closed proximity could interfere and resulting in loss of valuable information that could be devastating when diagnosed with partial data. We propose a highly flexible and versatile reconfigurable hybrid optical-radio wireless communication network for both stationary and ambulatory healthcare. We present two cases here; the former case explains the proposed hybrid optical-radio network within a RF sensitive facility in $\mathrm{HoF}$ and the later case describes the extension of WBAN to ROWBAN for in hospital and remote healthcare monitoring.

\subsection{Hybrid Wireless Communications in RF Sensitive Areas}

RF communication exposes radiation to human and in certain facilities within hospital RF communication is not favorable, generally where highly charged electromagnetic devices are installed. Examples include MRI labs, treatment facilities of nuclear medicine and radiation therapy facilities, where the dense shielding and bunker walls do not favor RF. During the procedures, the patients are examined with several lifesaving medical devices/sensors e.g., electrocardiography (ECG), electroencephalography (EEG) and non-invasive blood pressure (NIBP) within these facilities. The data from these lifesaving devices is examined continuously and stored remotely. In case of no RF, the reconfigurable hybrid optical-radio network selects the Mode 1 and the data from these lifesaving sensing devices can be transmitted on VLC. During procedures the amount of produced data by these devices is usually quite large, thus a high link speed is required to transport the data to the servers. VLC supports high throughput [45] and can transmit bulk data. In these facilities, the latency of the wireless link can be high for transporting data to the servers for diagnostics. Offloading can be done in case of huge sensors' network i.e., transmitting data to servers after procedure is completed. Figure 3 shows the picture of procedure room connected to a hybrid network, where a patient is being examined and data from numerous supporting sensing devices and main examination device is transmitted on VLC.

\subsection{Hybrid Wireless Communications for Ambulatory and Home Care}

The reconfigurable radio-optical wireless body area network ROWBAN is an extension of conventional WBAN, adding 


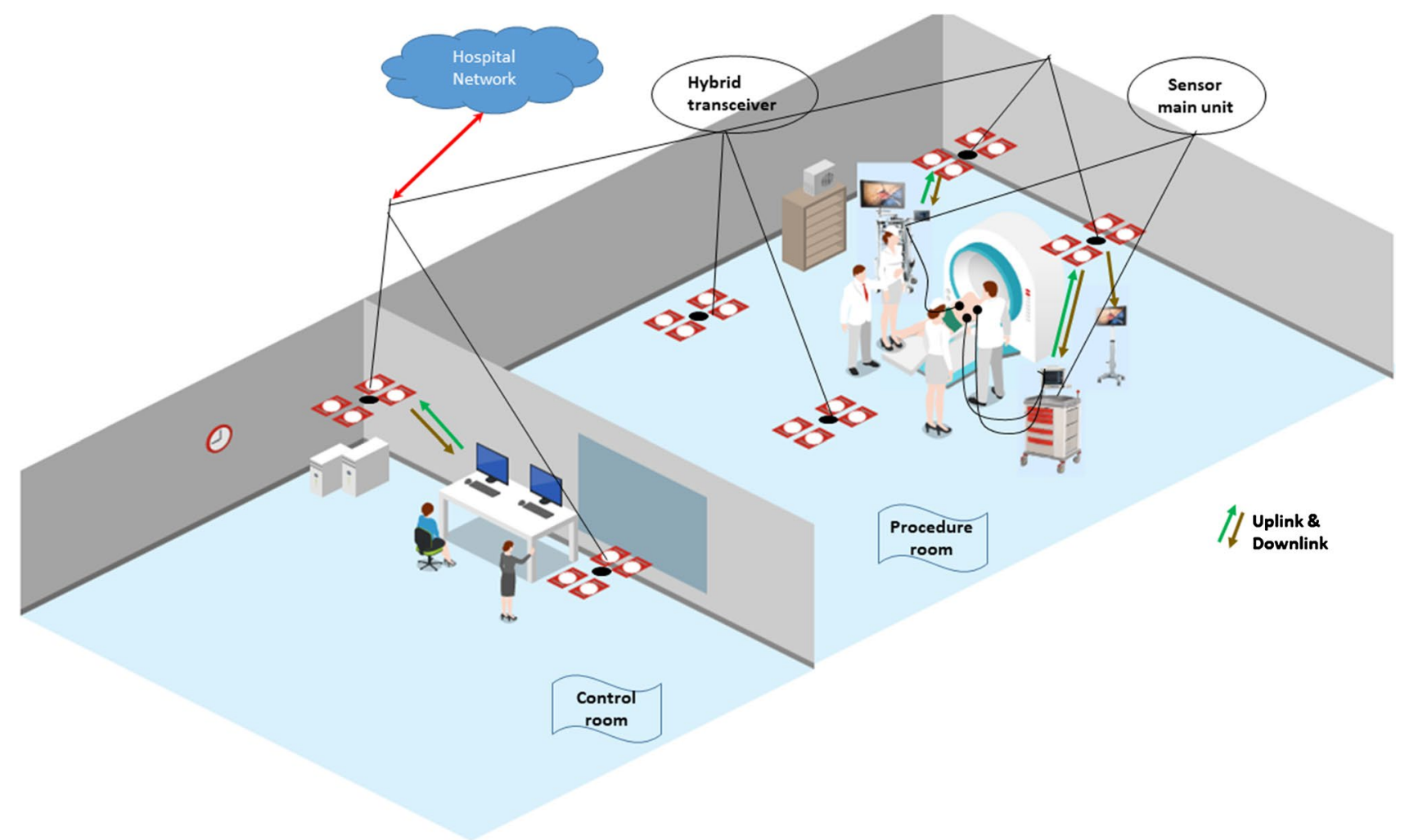

Fig. 3 OWC in RF sensitive area. An overview of a procedure room where number of sensing devices are connected to a patient and data is being observed in real-time to make decisions

optical nodes to the existing WBAN mesh to make it more generic for HoF. The ROWBAN connects to an AP to share information as shown in Fig. 4. The concept of AP is analyzed in our previous works $[43,46]$ and the optical-radio nodes can be reconfigured dynamically to operate in any of the five modes described earlier. ROWBAN can have $k$ nodes, it may eventually contain all the coexisting hybrid nodes or in some scenarios $i$ nodes out of $k$ can be coexisting

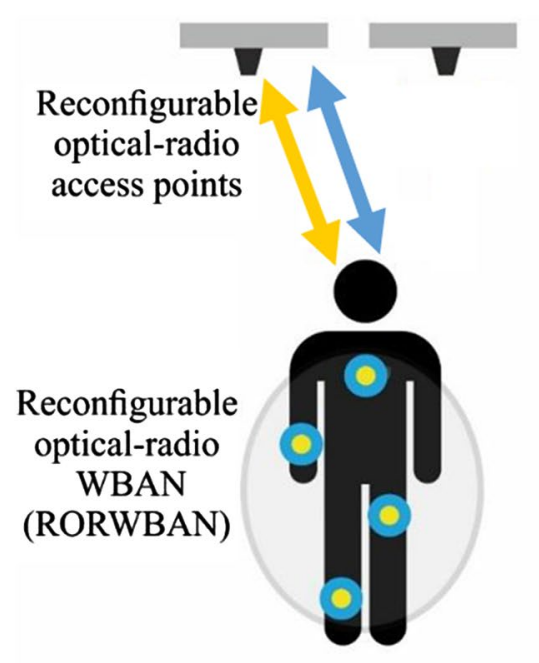

Reconfigurable optical-radio wireless networks
for the hospital of the future

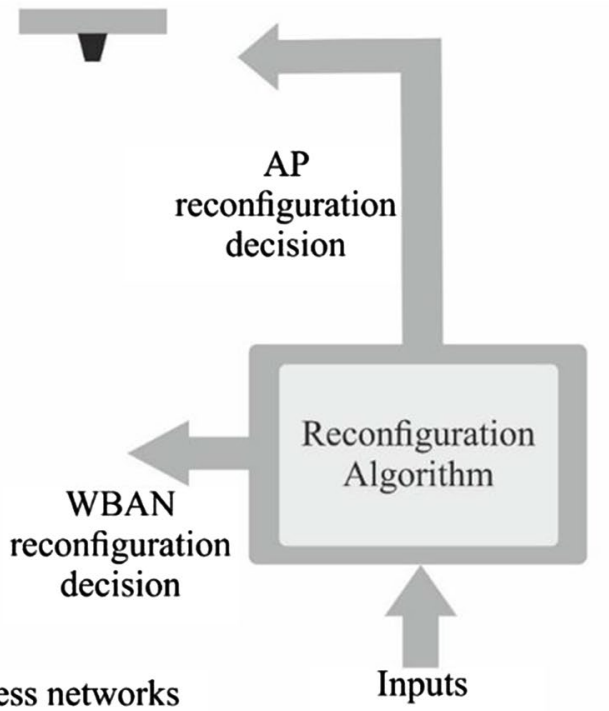

Inputs

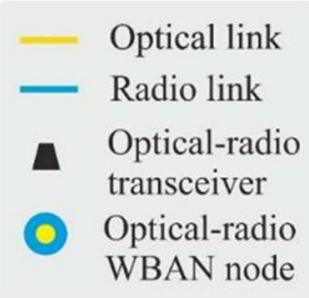

Algorithm inputs

- Type of scenario

- Channel condition

- Local policies

- Service provider

decisions

- User decisions

- Etc.

Fig. 4 Reconfigurable optical-radio access points and ROWBAN 
i.e., containing both optical-radio transceivers and the rest $(k-i)$ contain single technology. The term reconfigurable in ROWBAN reflects its dynamic nature that can be configure complying both long term changes i.e., user policies or user requirements as dictated and certain instantaneous changes e.g., blockage, channel degradation etc. The overall goal of reconfiguration is to make our proposed system flexible to adopt changes and in certain cases user can implement its own policies to meet own requirement. We studied a single coexisting node for ROWBAN, the data from patient is sent to an AP within the hospital, the selection of appropriate mode is performed by intelligent algorithm. In case of Mode 1 , when a patient comes in contact with AP the data transmission starts immediately.

The algorithm observes the instantaneous conditions and establishes the UL and DL channels complying the conditions according to the predefined profile stored in algorithm. An important profile for mode selection in ROWBAN is utilizing the power datagram, data transmission is done utilizing one of the five mode that consumes minimum power.

Remote healthcare is getting more attention nowadays as for chronic patients the self-monitoring of chronic indicators such as blood sugar, blood pressure, heart rate etc., could strengthen them to cure their disease. The concept of $\mathrm{HoF}$ is to provide healthcare services beyond the hospital premises, converting home into remote healthcare facilities and connecting them with HoF. ROWBAN provides a viable solution to monitor several chronic health factors, no matter the patient is stationary or moving, the hybrid optical-radio nodes can play a vital role in transmitting data to the cloud or HoF. With these hybrid nodes, the possible data losses due to electromagnetic interference could be overcome by using optical networks. Figure 4 shows the proposed architecture of ROWBAN and an AP to receive sensors' data. In $\mathrm{HoF}$, the reliability and security of data from ambulatory or home hospital is of great concerns and have given top priority as in Table 1, since the data is transmitted from a distant location the interference or intentional hampering of data can affect the diagnosis. The integrity of wireless network is an important factor and should possess high integrity to ensure patient's health.

\section{Challenges}

Here we will discuss challenges related to visible light technology and hybrid communication (optical + RF) system development to fulfil the desired requirements.

Security features must span across the whole hybrid communication system. For optical-only based communication technology the challenges related to security features implementation are mainly connected with reliability, namely with the need for a LOS channel between the transmitter and in order to achieve maximum data throughput. If the LOS condition is not available, the data connection might support lower data rate, be less reliable or eventually the link could be interrupted. This will affect mainly on the wireless connection dependability, whereas the aspects of patient privacy and low SAR exposure will not be jeopardized.

However, with a hybrid system, the challenges are more complex. In cases when the LOS condition for the VLC is not available, RF technologies will be used. In such cases, to ensure seamless communication devising a smooth handover mechanism exposes challenges in Open Systems Interconnection (OSI) model. Hybrid system is proposed to enhance the transmission, packet loss is not necessary inevitable if the packets will be buffered, and then transmitted once the handover has been made. But for sure, some delay is inevitable. An extensive approach is required to gather the accurate CSI for both optical and RF channels in order to make decision on link deterioration beforehand and to perform instant switching to avoid packet loss [47]. As in Figs. 2 and 3, our proposed $\mathrm{HoF}$ forms a mesh of hybrid APs, a single user can be connected to multiple APs could be beneficial in enhancing the transmission by keeping most powerful link alive. But for mobile user performing handover not only between different optical APs as well as RF is key. As the user moves from one cell to another the CSI determines a great role in making handover decisions and a robust decision making algorithm is key for higher performances. The optical-radio hotspots form a small coverage cells, as in [48] author simulated overlapping and non-overlapping small coverage cells formed by hybrid network, the throughput of overlapping hybrid cells are found to be maximum but the load balancing is clearly a challenge in order to maximize the throughput in crowded hybrid network.

From the security point of view, the data is exposed to leakage to neighboring premises, and also to interference originating from them. The SAR load to humans will be increased as well. In [49], author presented a feasibility for eavesdropping in VLC network. The author presented that VLC signal can be jammed locally in large coverage area as well as the illegal transmission can be introduced from outside. The current study raises concerns over the security in VLC. However the parallel optical-radio transmission can be beneficial solution to enhance the network's physical layer security when intruded by eavesdropper [50].

Seamless communication system which takes advantage of optical and RF communication 696 must designed to be intelligent by using machine learning (ML) and AI in the decision making. By collecting information about the network nodes behavior and communication related parameters, ML and AI can optimize the hybrid system performance. However, optimization algorithm development for this task is very complex and challenging since hybrid system includes various type of devices with 
Table 3 Suitability of wireless technologies to the connected $\mathrm{HoF}$

\begin{tabular}{llllllll}
\hline & Inpatient care & $\begin{array}{l}\text { Ambulatory } \\
\text { care }\end{array}$ & $\begin{array}{l}\text { Diagnostic/ } \\
\text { treatment }\end{array}$ & $\begin{array}{l}\text { Support } \\
\text { services }\end{array}$ & Public spaces & $\begin{array}{l}\text { Outpatient care } \\
\text { (home) } \\
\text { Outpatient } \\
\text { care (on the } \\
\text { move) }\end{array}$ \\
\hline Radio & $3-4$ & $3-4$ & $2-3$ & $4-5$ & $4-5$ & $3-4$ & $4-5$ \\
Optical & $3-5$ & $3-5$ & $4-5$ & $4-5$ & $2-3$ & $3-5$ & $1-2$ \\
Optical + Radio & $4-5$ & $4-5$ & $4-5$ & $4-5$ & 3 & $4-5$ & $2-3$ \\
\hline
\end{tabular}

5: very-high; 4: high; 3 : moderate, 2 : low; 1 : very-low

different needs. Network control should be done in realtime without sacrificing the communication performance, especially in case of critical medical data transfer.

Novel energy harvesting solutions are very important to enable energy efficiency of future communication solutions. In [51, 52], authors proposed to harvest energy for sensors from LED transmitter. Ultimate target is to design energy neutral sensor solutions as much as possible, i.e., sensor nodes would harvest the required energy from their operation environment. It is very challenging to design and implement enough efficient energy harvesting which operates in tiny sensor nodes. Visible light system could be used to also provide energy that would be harvested by ultra-low power sensor devices.

Certain medical examinations require high throughput which is challenging to achieve in congested RF bands. $5 \mathrm{G}$ and beyond systems are moving to higher frequencies to achieve higher throughput which will ease the problem. However, it will be advantageous to design the hybrid solutions which takes advantage of very high throughput offered by visible light communication. With VLC high data rates up to $1 \mathrm{Gbit} / \mathrm{s}$ have already been reported [45], making it suitable as an answer to the use cases in the future hospital with the highest demands of data rates. However, in cases with non-LOS conditions, VLC may not be available. Hybrid systems should operate in a seamless way to select the best technology in real-time to offer required communication performance without sacrificing security and reliability.

Management and operation of hybrid system should be designed by keeping the reconfiguration complexity in mind. Otherwise the tradeoff between achieved gains and increased complexity will turn negative. Reconfiguration complexity may also lead to increased communication latency if the decision making takes too much time. The changing operational environments related to, e.g., the visible light channel state set challenges affecting to the complexity of the reconfigurable hybrid RF/system. Depending on the situation, sometimes a high data rate is the main priority, in some cases it may be, e.g., the low latency, security or reliability.

\section{Discussions}

Connectivity empowers a hospital, making its operation more flexible and efficient. It is noted that the notion of "hospital", as used and understood today, may evolve in the future. As in [53], a pervasive healthcare solution is proposed, where clinicians, nurses, subjects and other relevant people are connected through reliable wireless networks, as well as specialized treatment is provided in distributed points too. In fact, as a result of the increasing ubiquity and performance of wireless and mobile networks, the concept of hospital as a well-defined place where patients receive medical treatment is likely to evolve as a pervasive healthcare. Wireless connectivity provided with conventional radio technologies has unique advantages and it is expected that will radio communications be an integral technology supporting the concept of HoF. OWC particularly VLC, is a complementary approach to radio, being particularly attractive in scenarios where radio fails to provide security, privacy and zero electromagnetic radiation. The combination of both technologies in an integrated manner is an unmatched solution, in terms of performance, flexibility, scalability, security, privacy, interference and exposure. In order to fully exploit these advantages, connected nodes as well as APs of a hospital need to be designed to support hybrid radio-optical networks, a departure from today's radio-only approach. Based on Table 1 and the discussions on the advantages of the considered wireless network, Table 3 illustrates the suitability of radio-, optical- and hybrid network to the key scenarios of a HoF. As can be observed, the combination of both access technologies is highly suitable in most of the hospital scenarios.

In terms of current developments of future communication networks, $5 \mathrm{G}$ networks are about to be deployed and expected to be highly used in the 2020-2030 decade. Moreover, 6G development will take place during the mentioned decade [54]. 5G represents a radio-only technology while $6 \mathrm{G}$ is considered an integrated communication platform encompassing multiple frequency bands (in the low $\mathrm{GHz}$ bands, $\mathrm{mm}$-waves and $\mathrm{THz}$ bands), including 
in the upper extreme light-based communications. Veryhigh frequency bands as well as light are suitable mostly for short-range communications, therefore relevant to the indoor-environments of the HoF. 6G, expected to be available commercially from 2030 on, will provide a complete solution for the connected $\mathrm{HoF}$, as it will encompass a flexible platform using different spectum bands and technologies that can be selected and optimized on the fly to accomplish a specific task. In fact, such a universal virtualized software-defined network will chose, combine and optimize technologies (e.g., modulation, coding, use of multidimensional resources, etc.) and transmission domains (radio bands, light wavelengths) to carry out a communication session with a number of requirements dictated by the type of service, scenario, etc. Such integrated system, working under centralized or distributed management appears to be a highly attractive solution for the HoF.

\section{Conclusion}

This paper considers wireless and mobile communications for the hospital of the future. The HoF is explained, in particular from the standpoint of its communications. Communicational requirements for the key scenarios of the $\mathrm{HoF}$ are introduced and discussed. Both radio and optical wireless networks are considered. Both network approaches have unique advantages, but no single network is best suitable for all the scenarios of the HoF. Therefore, we proposed a reconfigurable hybrid optical-radio network as the key backbone network for the HoF. Such network is discussed and its suitability to relevant scenarios is discussed. Moreover, a reconfigurable optical-radio body area network (ROWBAN) is introduced as the counterpart network to the reconfigurable hybrid access network. The solution exploits the advantages of both network approaches and is a future-proof solution for advanced healthcare in the years to come. Future work include more precise characterization of the requirements of the environments, as well as implementing and evaluating the performance of a reconfigurable hybrid network particularly suitable for healthcare scenario.

\footnotetext{
Acknowledgements Open access funding provided by University of Oulu including Oulu University Hospital. Authors would like to thank the Academy of Finland's support in the project HERONET, where this research work was mainly carried out. In addition, the support from the Academy of Finland 6Genesis Flagship Project is warmly acknowledged.
}

Open Access This article is distributed under the terms of the Creative Commons Attribution 4.0 International License (http://creativeco mmons.org/licenses/by/4.0/), which permits unrestricted use, distribution, and reproduction in any medium, provided you give appropriate credit to the original author(s) and the source, provide a link to the Creative Commons license, and indicate if changes were made.

\section{References}

1. J. Ehrich, E. Molloy, R. Kerbl, M. Pettoello-Mantovani and A. Gerber-Grote, Conceptual design of future children's hospitals in Europe. The role of public and private stakeholders as transferors of new concepts from theory into practice, Journal of Pediatrics, Vol. 183, pp. 1-2, 2017.

2. J. D. Zajac, The public hospital of the future, Medical Journal of Australia, Vol. 179, No. 5, pp. 250-252, 2003.

3. M. Hensher, N. Edwards and R. Stokes, International trends in the provision and utilisation of hospital care, BMJ, Vol. 319, No. 7213, pp. 845-848, 1999.

4. A. M. Mokhtar, The future hospital: a business architecture view, The Malaysian Journal of Medical Sciences: MJMS, Vol. 24, No. 5, p. 1, 2017.

5. http://www.oys2030.fi/english.php. Accessed 26 June 2019.

6. S. Landers, E. Madigan, B. Leff, R. J. Rosati, B. A. McCann, R. Hornbake and T. Lee, The future of home health care: a strategic framework for optimizing value, Home Health Care Management \& Practice, Vol. 28, No. 4, pp. 262-278, 2016.

7. G. López, V. Custodio and J. I. Moreno, LOBIN: E-textile and wireless-sensor-network-based platform for healthcare monitoring in future hospital environments, IEEE Transactions on Information Technology in Biomedicine, Vol. 14, No. 6, pp. 1446-1458, 2010.

8. W. Noonpakdee, Adaptive wireless optical transmission scheme for health monitoring system, in 2013 IEEE Third International Conference on Consumer Electronics? Berlin (ICCE-Berlin), IEEE, pp. 161-64, 2013.

9. R. Murai, T. Sakai, H. Kawano, Y. Matsukawa, Y. Kitano, Y. Honda, and K. C. Campbell, A novel visible light communication system for enhanced control of autonomous delivery robots in a hospital, in 2012 IEEE/SICE International Symposium on System Integration (SII), IEEE, pp. 510-6, 2012.

10. A. Heitmann, R. S. Cišić and I. Meyenburg-Altwarg, From magnet-hospital to the hospital of the future, Nursing and Health, Vol. 1, No. 4, pp. 78-87, 2013.

11. J. E. Bardram, Hospitals of the future-ubiquitous computing support for medical work in hospitals. in Proceedings of UbiHealth, Vol. 3. 2003.

12. C. X. Wang, F. Haider, X. Gao, X. H. You, Y. Yang, D. Yuan and E. Hepsaydir, Cellular architecture and key technologies for $5 \mathrm{G}$ wireless communication networks, IEEE Communications Magazine, Vol. 52, No. 2, pp. 122-130, 2014.

13. W. Ding, F. Yang, H. Yang, J. Wang, X. Wang, X. Zhang and J. Song, A hybrid power line and visible light communication system for indoor hospital applications, Computers in Industry, Vol. 68, pp. 170-178, 2015.

14. J. Song, W. Ding, F. Yang, H. Yang, J. Wang, X. Wang, and X. Zhang, Indoor hospital communication systems: an integrated solution based on power line and visible light communication. in 2014 IEEE Faible Tension Faible Consommation, IEEE, pp. 1-6, 2014.

15. C. Huang and X. Zhang, Impact and Feasibility of Darklight LED on Indoor visible light positioning system, in 2017 IEEE 17th International Conference on Ubiquitous Wireless Broadband (ICUWB), IEEE, pp. 1-5, 2017.

16. H. Thimbleby, Technology and the future of healthcare. Journal of Public Health Research, Vol. 2, No. 3, 2013.

17. L. Taylor, Hospitals of the future, British Medical Journal, Vol. 2, No. 5201, p. 752, 1960. 
18. J. Ribera, G. Antoja, and M. Rosenmöller, Hospital of the future: a new role for leading hospital in Europe, 2016.

19. D. Seth, and D. Chakraborty, Hidden Markov model and Internet of Things hybrid driven smart hospital. in 2017 8th International Conference on Computing, Communication and Networking Technologies (ICCCNT), IEEE, pp. 1-7, 2017.

20. P. K. Sahoo, S. K. Mohapatra and S. L. Wu, Analyzing healthcare big data with prediction for future health condition, IEEE Access, Vol. 4, pp. 9786-9799, 2016.

21. H. Alemdar and C. Ersoy, Wireless sensor networks for healthcare: a survey, Computer Networks, Vol. 54, No. 15, pp. 2688$2710,2010$.

22. A. Mainwaring, D. Culler, J. Polastre, R. Szewczyk, and J. Anderson, Wireless sensor networks for habitat monitoring. in Proceedings of the 1st ACM International Workshop on Wireless Sensor Networks and Applications, ACM, pp. 88-97, 2002.

23. V. Jones, R. Bults, D. Konstantas, and P. Vierhout, Healthcare PANs: Personal Area Networks for trauma care and home care, 2001.

24. S. Ullah, H. Higgins, B. Braem, B. Latre, C. Blondia, I. Moerman and K. S. Kwak, A comprehensive survey of wireless body area networks, Journal of medical Systems, Vol. 36, No. 3, pp. 1065-1094, 2012

25. H. Zhang, J. Li, B. Wen, Y. Xun and J. Liu, Connecting intelligent things in smart hospitals using NB-IoT, IEEE Internet of Things Journal, Vol. 5, No. 3, pp. 1550-1560, 2018.

26. M. S. Saud, I. Ahmed, T. Kumpuniemi, and M. Katz, Reconfigurable optical-radio wireless networks: Meeting the most stringent requirements of future communication systems. Transactions on Emerging Telecommunications Technologies, Vol. 30, No. 2, 2019.

27. I. Ahmed, T. Kumpuniemi and M. Katz, A Hybrid OpticalRadio Wireless Network Concept for the Hospital of the Future, BodynetsOulu, 2018.

28. H. Karvonen, M. Hämäläinen, J. Iinatti, and C. Pomalaza-Ráez, Coexistence of wireless technologies in medical scenarios, in 2017 European Conference on Networks and Communications (EuCNC), IEEE, pp. 1-5, 2017.

29. H. Karvonen, K. Mikhaylov, M. Hämäläinen, J. Iinatti, and C. Pomalaza-Ráez, Interference of wireless technologies on BLE based WBANs in hospital scenarios, in 2017 IEEE 28th Annual International Symposium on Personal, Indoor, and Mobile Radio Communications (PIMRC), IEEE, pp. 1-6, 2017.

30. H. Karvonen, K. Mikhaylov, D. Acharya and R. Moklesur, Performance Evaluation of Bluetooth Low Energy Technology under Interference, BodynetsOulu, 2018.

31. R. Sprow, Planning Hospitals of the Future. Designing Hospitals of the Future, Prism PublicationsMumbai, 2012.

32. W. Sun, Z. Cai, Y. Li, F. Liu, S. Fang, and G. Wang, Security and privacy in the medical internet of things: a review. in Security and Communication Networks, 2018.

33. M. Al Ameen, J. Liu and K. Kwak, Security and privacy issues in wireless sensor networks for healthcare applications, Journal of Medical Systems, Vol. 36, No. 1, pp. 93-101, 2012.

34. M. Kashef, A. Torky, M. Abdallah, N. Al-Dhahir, and K. Qaraqe, On the achievable rate of a hybrid PLC/VLC/RF communication system. in 2015 IEEE Global Communications Conference (GLOBECOM), IEEE, pp. 1-6, 2015.

35. S. I. Hussain, M. M. Abdallah, and K. A. Qaraqe, Hybrid radiovisible light downlink performance in RF sensitive indoor environments. in 2014 6th International Symposium on Communications, Control and Signal Processing (ISCCSP), IEEE, pp. 81-4, 2014.

36. M. Kashef, M. Ismail, M. Abdallah, K. A. Qaraqe and E. Serpedin, Energy efficient resource allocation for mixed RF/VLC heterogeneous wireless networks, IEEE Journal on Selected Areas in Communications, Vol. 34, No. 4, pp. 883-893, 2016.

37. Z. Huang, Z. Wang, M. Huang, W. Li, T. Lin, P. He and Y. Ji, Hybrid optical wireless network for future SAGO-integrated communication based on FSO/VLC heterogeneous interconnection, IEEE Photonics Journal, Vol. 9, No. 2, pp. 1-10, 2017.

38. W. A. Cahyadi, T. I. Jeong, Y. H. Kim, Y. H. Chung, and T. Adiono, Patient monitoring using visible light uplink data transmission. in 2015 International Symposium on Intelligent Signal Processing and Communication Systems (ISPACS), IEEE, pp. 431-4, 2015.

39. D. R. Dhatchayeny, A. Sewaiwar, S. V. Tiwari, and Y. H. Chung EEG biomedical signal transmission using visible light communication, in 2015 International Conference on Industrial Instrumentation and Control (ICIC), IEEE, pp. 243-246, 2015.

40. M. Kafafy, Y. Fahmy, M. Abdallah, and M. Khairy, Power efficient downlink resource allocation for hybrid RF/VLC wireless networks. in 2017 IEEE Wireless Communications and Networking Conference (WCNC), IEEE, pp. 1-6, 2017.

41. X. Wu, D. Basnayaka, M. Safari, and H. Haas, Two-stage access point selection for hybrid VLC and RF networks. in 2016 IEEE 27th Annual International Symposium on Personal, Indoor, and Mobile Radio Communications (PIMRC), IEEE, pp. 1-6, 2016.

42. R. Liu, and C. Zhang, Dynamic dwell timer for vertical handover in VLC-WLAN heterogeneous networks, in 2017 13th International Wireless Communications and Mobile Computing Conference (IWCMC), IEEE, pp. 1256-60, 2017.

43. M. S. Saud, and M. Katz, Implementation of a hybrid opticalRF wireless network with fast network handover, in European Wireless 2017; 23th European Wireless Conference, VDE, pp. $1-6,2017$.

44. S. Liang, H. Tian, B. Fan, and R. Bai, A novel vertical handover algorithm in a hybrid visible light communication and LTE system. in 2015 IEEE 82nd Vehicular Technology Conference (VTC2015-Fall), IEEE, pp. 1-5, 2015.

45. G. Cossu, W. Ali, R. Corsini and E. Ciaramella, Gigabit-class optical wireless communication system at indoor distances (1.5-4 m), Optics Express, Vol. 23, No. 12, pp. 15700-15705, 2015.

46. M. S. Saud, H. Chowdhury, and M. Katz, Heterogeneous software-defined networks: implementation of a hybrid radio-optical wireless network. in 2017 IEEE Wireless Communications and Networking Conference (WCNC), IEEE, pp. 1-6, 2017.

47. M. Ayyash, H. Elgala, A. Khreishah, V. Jungnickel, T. Little, S. Shao and R. Freund, Coexistence of WiFi and LiFi toward 5G: concepts, opportunities, and challenges, IEEE Communications Magazine, Vol. 54, No. 2, pp. 64-71, 2016.

48. X. Li, R. Zhang and L. Hanzo, Cooperative load balancing in hybrid visible light communications and $\mathrm{WiFi}$, IEEE Transactions on Communications, Vol. 63, No. 4, pp. 1319-1329, 2015.

49. G. Blinowski, The feasibility of launching physical layer attacks in visible light communication networks, 2016, arXiv preprint arXiv: 1608.07146.

50. M. F. Marzban, M. Kashef, M. Abdallah, and M. Khairy, Beamforming and power allocation for physical-layer security in hybrid RF/VLC wireless networks. in 2017 13th International Wireless Communications and Mobile Computing Conference (IWCMC), IEEE, pp. 258-63, 2017.

51. T. Rakia, H. C. Yang, F. Gebali, and M. S. Alouini, Dual-hop VLC/RF transmission system with energy harvesting relay under delay constraint. in 2016 IEEE Globecom Workshops (GC Wkshps), IEEE, pp. 1-6, 2016.

52. G. Pan, H. Lei, Z. Ding, and Q. Ni, On 3-D hybrid VLC-RF systems with light energy harvesting and OMA scheme over 
RF links. in GLOBECOM 2017-2017 IEEE Global Communications Conference, IEEE, pp. 1-6, 2017.

53. U. Varshney, Pervasive healthcare, Computer, Vol. 36, No. 12, pp. 138-140, 2003.

54. https://www.oulu.fi/6gflagship/. Accessed 26 June 2019.

Publisher's Note Springer Nature remains neutral with regard to jurisdictional claims in published maps and institutional affiliations.

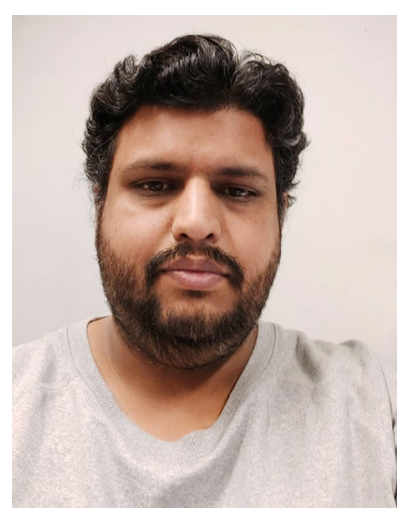

Iqrar Ahmed is currently a $\mathrm{PhD}$ student at University of Oulu.

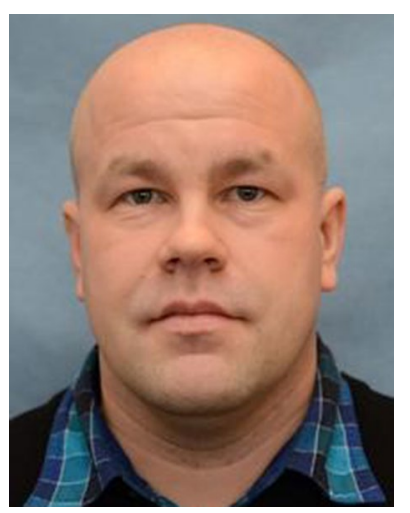

Dr. Heikki Karvonen is research fellow at University of Oulu.

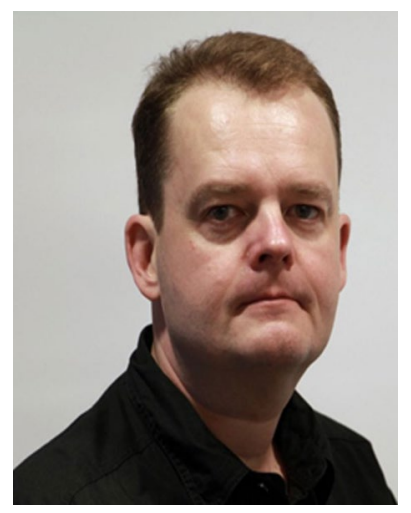

Timo Kumpuniemi is doctoral student at University of Oulu.

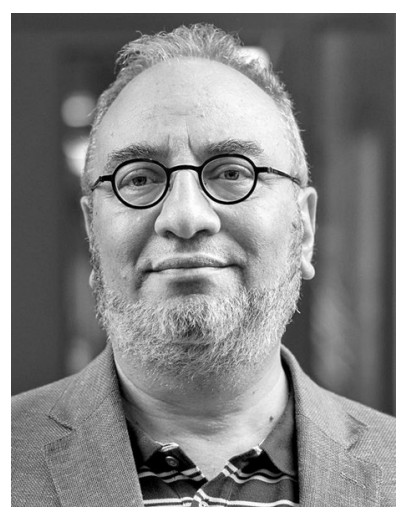

Dr. Marcos Katz is a professor at University of Oulu. 